\title{
Pathological complete response to neoadjuvant chemotherapy, but not the addition of carboplatin, is associated with improved survival in Chilean triple negative breast cancer patients: a report of real world data
}

\author{
Benjamín Walbaum ${ }^{1}$, Francisco Acevedo ${ }^{1}$, Lidia Medina ${ }^{2}$, M Loreto Bravo ${ }^{1}$, Tomas Merino ${ }^{1}$, Mauricio Camus ${ }^{3}$, Francisco Domin- \\ guez $^{3}$, Sebastián Mondaca ${ }^{1}$, Héctor Galindo ${ }^{1}$, Bruno Nervi ${ }^{1}$, Carolina Ibañez ${ }^{1}$, Jorge Madrid ${ }^{1}$, Sabrina Muñiz ${ }^{4}$, José Peña ${ }^{1}$, Érica Koch ${ }^{1}$, \\ Marcelo Garrido ${ }^{1}$, Mauricio P Pinto ${ }^{1}$ and César Sánchez ${ }^{1}$

\begin{abstract}
${ }^{1}$ Department of Hematology-Oncology, Faculty of Medicine, Pontificia Universidad Católica de Chile, Diagonal Paraguay 362, Santiago, Chile ${ }^{2}$ Cancer Center 'Nuestra Señora de la Esperanza', Red de Salud UC Christus, Pontificia Universidad Católica de Chile, Santiago, Chile ${ }^{3}$ Department of Surgical Oncology, Faculty of Medicine, Pontificia Universidad Católica de Chile, Santiago, Chile ${ }^{4}$ Complejo Asistencial Hospital Dr Sotero del Rio, Santiago, Chile
\end{abstract}

\section{Abstract}

Background: Breast cancer (BC) is the leading cause of cancer death for Chilean women. About $11 \%$ of cases are triple-negative (TN) BC. These are characterised by poor prognosis, higher risk of early recurrence and visceral dissemination versus other BC subtypes. Current standard treatment for early-stage non-metastatic TNBC patients consists of neoadjuvant chemotherapy (NACT) followed by surgery and radiotherapy. Pathological complete response ( $p C R$ ) to NACT is associated with an increase in survival rates. In general, NACT and adjuvant regimens involve similar cytotoxic drugs. Recent studies have postulated that the use of platinum compounds in TNBC would increase response rates. However, their effects on patient survival remain uncertain.

Materials and methods: We retrieved and analysed medical records from a total of 156 Chilean stage I-III TNBC female patients that received NACT and compared survival rates using carboplatin $(\mathrm{Cb})$-containing versus non- $\mathrm{Cb}$-containing regimens at two health cancer centres.

Results: Median age was 51 years (range: $24-81) ; 13.5 \%(n=21)$ received $\mathrm{Cb}$-containing regimens, $80.1 \%(n=125)$ received sequential anthracyclines plus taxanes; $29.5 \%(n=$ 46) of the total group achieved pCR, $28 \%$ for the standard treatment and $35 \%(n=8)$ for the $\mathrm{Cb}$-containing group $(p=0.59)$. We confirmed $\mathrm{pCR}$ was associated with prolonged overall survival, invasive and distant disease-free survival (Log-rank $p=0.0236$ ). But the addition of $\mathrm{Cb}$ was not associated with differences in survival measures (Log-rank $p=$ 0.5216).

Conclusions: To the best of authors' knowledge, this is the first report on real-world data in the Chilean population assessing the effect of $\mathrm{Cb}$-containing NACT in TNBC. The authors' results suggest no survival benefit by the addition of $\mathrm{Cb}$ to standard NACT. However, we confirm an increase in survival associated to pCR regardless of treatment.

Keywords: breast neoplasm, triple negative, neoadjuvant, chemotherapy, carboplatin

Correspondence to: César Sánchez Email: csanchez@med.puc.cl

ecancer 2021, 15:1178

https://doi.org/10.3332/ecancer.2021.1178

Published: 01/02/2021

Received: 08/09/2020

Publication costs for this article were supported by ecancer (UK Charity number 1176307).

Copyright: (c) the authors; licensee ecancermedicalscience. This is an Open Access article distributed under the terms of the Creative Commons Attribution License (http:// creativecommons.org/licenses/by/3.0), which permits unrestricted use, distribution, and reproduction in any medium, provided the original work is properly cited. 


\section{Background}

Breast cancer (BC) is the leading cause of cancer death for Chilean women. Only in 2018, an estimated total of 5,400 women were diagnosed with BC causing 1,700 deaths [1]. Furthermore, recent reports in Western countries (including Chile) have revealed an increase in BC incidence [2-4]. These could be explained by a combination of factors such as changes in lifestyles, mainly associated with an ageing population, increased exposure to hormones and obesity rates along with better diagnostic methods.

Within this context, triple negative breast cancers (TNBCs) represent about $11 \%$ of cases in Chile. This subtype is characterised by its poor prognosis and higher recurrence rates versus other subtypes [5]. Current standard of care for stage I-III TNBC involves neoadjuvant chemotherapy (NACT) followed by surgical tumour removal. Indeed, NACT improves breast conservation levels in TNBC [6] plus in vivo assessment of therapy response. Nonetheless, these regimens have failed to improve survival compared to adjuvant chemotherapy (CT) [6]. Despite this, studies demonstrate that a post-NACT pathological complete response (pCR) is a key prognostic marker that defines post-surgery therapeutic strategies [7]. CT regimens usually include alkylating agents, taxanes and anthracyclines that achieve 40\% of pCR [7]; the addition of platinum compounds increases these percentages by 1.5 or 2 fold. Therefore, recent studies have proposed its addition to regular NACT schemes, however, given the added toxicity and the uncertain overall survival (OS) benefit, this is still controversial in management guidelines [8-10].

To date, the effect of platinum compounds on pCR or OS rates in Chilean TNBC patients remains unreported. Moreover, its usage is not incorporated into national management guidelines. Herein, we assessed pCR and survival rates in localised TNBC patients that received platinum-containing NACT at two cancer centres in the Santiago metropolitan area.

\section{Materials and methods}

\section{Study design and ethics approval}

This was a retrospective study conducted at two cancer centres: the Pontificia Universidad Católica de Chile and Dr. Sótero del Río hospitals. The Human Research Ethics Committee approved this study (approval number: \#200303006). All patient procedures strictly adhered to the principles of the Declaration of Helsinki (updated in 2013) and protected patient privacy according to ethical/legal standards.

\section{Patients and clinical data}

Clinical and epidemiological data were obtained from medical records. The authors' study included stage I-III TNBC patients selected for NACT at the Pontificia Universidad Catolica de Chile Cancer Center or the Dr. Sótero del Río hospital over the period 1997-2019. Hormone receptor status was inferred from pathology reports; TNBC was defined by ASCO-CAP criteria [11] as tumours that displayed <1\% positivity on nuclear staining for oestrogen or progesterone receptors in tumour cells and an immunoscore of 0 or $1+$ for human epidermal growth factor-type 2 receptor (HER2) or 2+ in the absence of amplification by fluorescent in situ hybridization (FISH). Clinical characteristics included NACT-platinum use, pCR rates defined as the absence of residual invasive disease on evaluation of the resected breast and lymph nodes after completing NACT (ypTO ypNO based on the American Joint Committee on Cancer staging system [12]).

\section{Statistical analysis}

Patients' OS were calculated from the date of diagnosis until the date of death or last follow-up (censored: August 2019). Similarly, Invasive Disease Free Survival (IDFS) was defined as the time from diagnosis until recurrence of invasive BC; Distant Disease Free Survival (DDFS) was defined as the time from diagnosis until systemic recurrence of BC. For the analysis of categorical variables, we used the chi-square test. The effects of systemic therapy regimens upon survival were assessed using the Kaplan-Meier method and the Log Rank test for equality of 
survivor functions. In cases where hazards were non-proportional (crossed survival curves), the Wilcoxon test was applied. Statistical significance was set at $p<0.05$. All data were analysed using STATA software version 16.1 (StataCorp LP, College Station, Texas, USA).

\section{Results}

A total of 156 stage I-III TNBC patients were analysed. Their main characteristics are summarised in Table 1. Median age at diagnosis was 51-year-old (range: 24-81) and the initial TNM stage distribution was 3\%, 44\% and 52\% for I, II and III; respectively. Six patients (3.8\%) had BRCA $1 / 2$ mutations, $80.7 \%$ received anthracycline plus taxane schemes and $13 \%$ had carboplatin (Cb) added to their regimens. Patients that received $\mathrm{Cb}$-containing NACT were predominantly from the private centre (66\%) and consequently $\mathrm{Cb}$ was more frequently used in this group compared to women at the public hospital ( $28 \%$ versus $8 \% ; p=0.046)$.

Patients in the $\mathrm{Cb}$ group tended to be younger versus non- $\mathrm{Cb}$ counterparts; however, this difference did not reach statistical significance (49-year-old versus 52-year-old, respectively; $p=0.34$ ). Additionally, no age differences were found for clinical stage, $\mathrm{pCR}$, death or recurrence rates. On average, $29 \%$ of the total of patients achieved pCR; $28 \%$ with the standard treatment and $35 \%$ in the group that received $\mathrm{Cb}$-containing NACT ( $p=0.59$, Table 1). Patients that did not receive sequential anthracyclines plus taxanes regimens (19.7\%) still received either anthracyclines or taxanes (75\%, $n=24$ and $22 \% n=7$; respectively) but displayed lower pCR rates (31.7\% versus 19\%; $p=0.17)$. When $\mathrm{Cb}$ use was excluded, this difference was even more evident ( $32 \%$ versus $13.7 \% ; p=0.052$ ).

On the other hand, a lower TNM BC stage was associated with an increase in pCR rates ( $40 \%$ for stage II versus $19 \%$ for stage III; $p=0.012$ ). At a 39-month median follow up, $22 \%$ of patients were deceased (35/156) and 28\% relapsed with invasive disease (43/154). Median OS was not reached for the entire analysed group (Figure 1a). As expected, stage III patients displayed a lower OS versus stages I or II (Log-rank test $p=0.0028$, Figure 1b). Next, we analysed survival rates in patients that displayed pCR. Indeed, these patients had a longer OS compared to patients without $\mathrm{pCR}$ (Log-rank $p=0.0236$, Figure 1c).

Finally, the addition of $\mathrm{Cb}$ to NACT did not modify OS (Log-rank $p=0.5216$, Figure $1 \mathrm{~d}$ ). In contrast, pCR and stage were associated with prolonged IDFS (Figure $2 \mathrm{a}-\mathrm{c}$ ) and DDFS (Figure $3 \mathrm{a}-\mathrm{c}$ ); whereas the addition of $\mathrm{Cb}$ had no significant impact in IDFS or DDFS (Figures $2 \mathrm{~d}$ and $3 \mathrm{~d}$ ).

Table 1. Clinical characteristics of 156 Chilean TNBC patients treated with NACT, with and without platinum.

\begin{tabular}{|l|c|c|c|c|}
\hline & CT without Cb & CT with Cb & Total & $p$-value \\
\hline Patients; $n$ (\%) & $136(87)$ & $20(13)$ & $156(100)$ & \\
\hline Average age (range) & $52(24-81)$ & $49(28-72)$ & $51(24-81)$ & 0.34 \\
\hline Stage; $n$ (\%) & & & & \\
I & $4(3)$ & $1(5)$ & $5(3)$ & 0.48 \\
II & $56(43)$ & $11(55)$ & $67(44)$ & \\
III & $71(54)$ & $8(40)$ & $79(52)$ & 0.59 \\
\hline pCR; $n$ (\%) & $38(28)$ & $7(35)$ & $45(29)$ & \\
\hline 3-year survival (\%) & & & & 0.52 \\
OS & $82(74-88)$ & $87(55-97)$ & $82(75-88)$ & 0.58 \\
DFS & $73(64-80)$ & $75(41-91)$ & $74(65-80)$ & 0.44 \\
DDFS & $74(65-81)$ & $83(48-96)$ & $75(66-81)$ & \\
\hline
\end{tabular}

CT; Chemotherapy, Cb; Carboplatin, pCR; Pathological complete response, OS; Overall survival, DFS; Disease-free survival, DDFS; Distant disease free survival

${ }^{a}$ Five patients missing data

b80 \% ( $n=125)$ sequential anthracycline plus taxane regimen, 15\% $(n=24)$ anthracyclines plus cyclophosphamides with or without fluorouracil and $4.5 \%(n=7)$ only paclitaxel. One patient received cyclophosphamide methotrexate and fluorouracil 

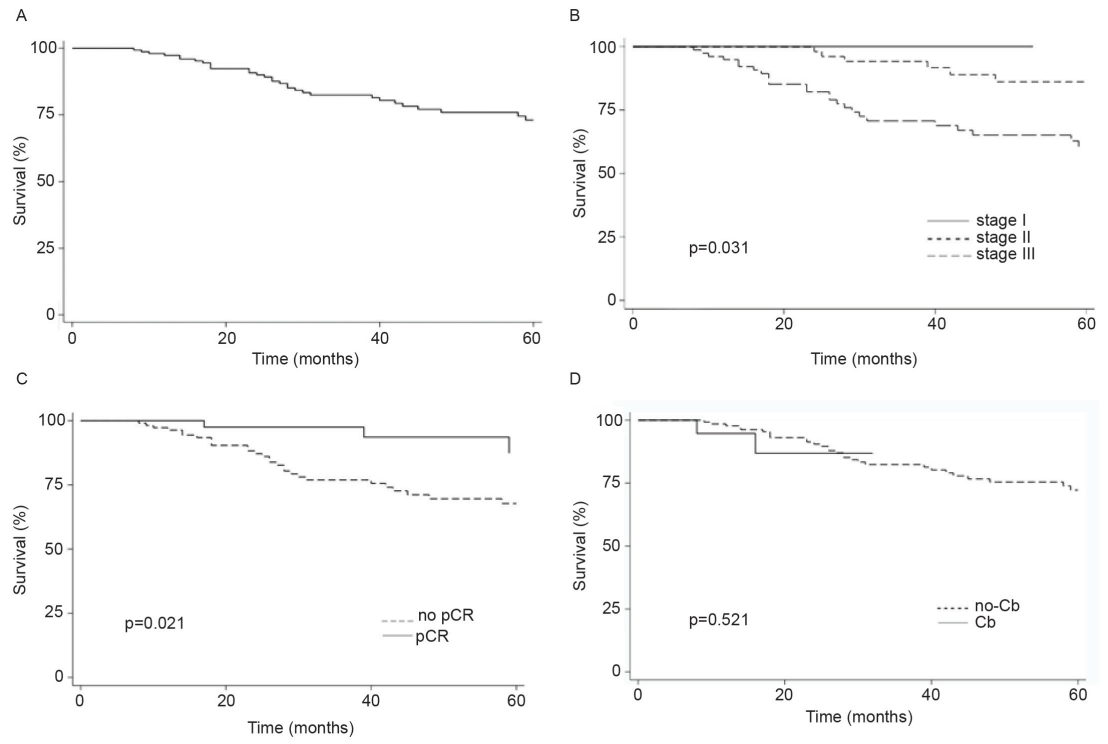

Figure 1. (a): OS in TNBC patients, (b): by clinical stage, (c): presence/absence of pCR or (d): carboplatin (Cb) use.
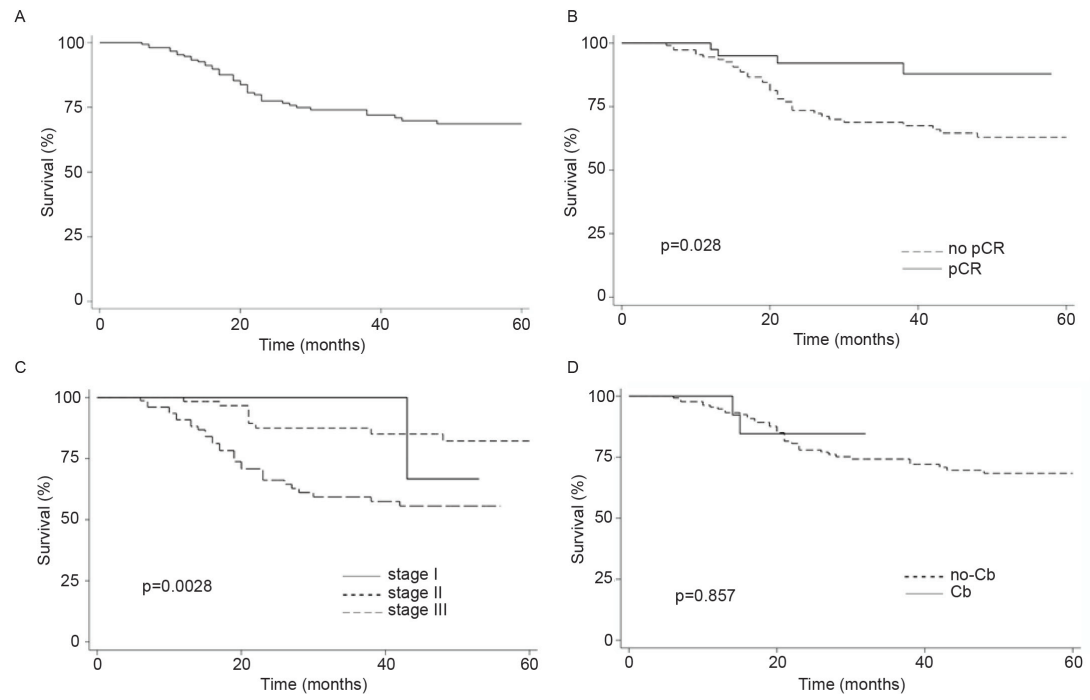

Figure 2. (a): IDFS in TNBC patients (b): by clinical stage, (c): presence/absence of pCR or (d): carboplatin (Cb) use. 

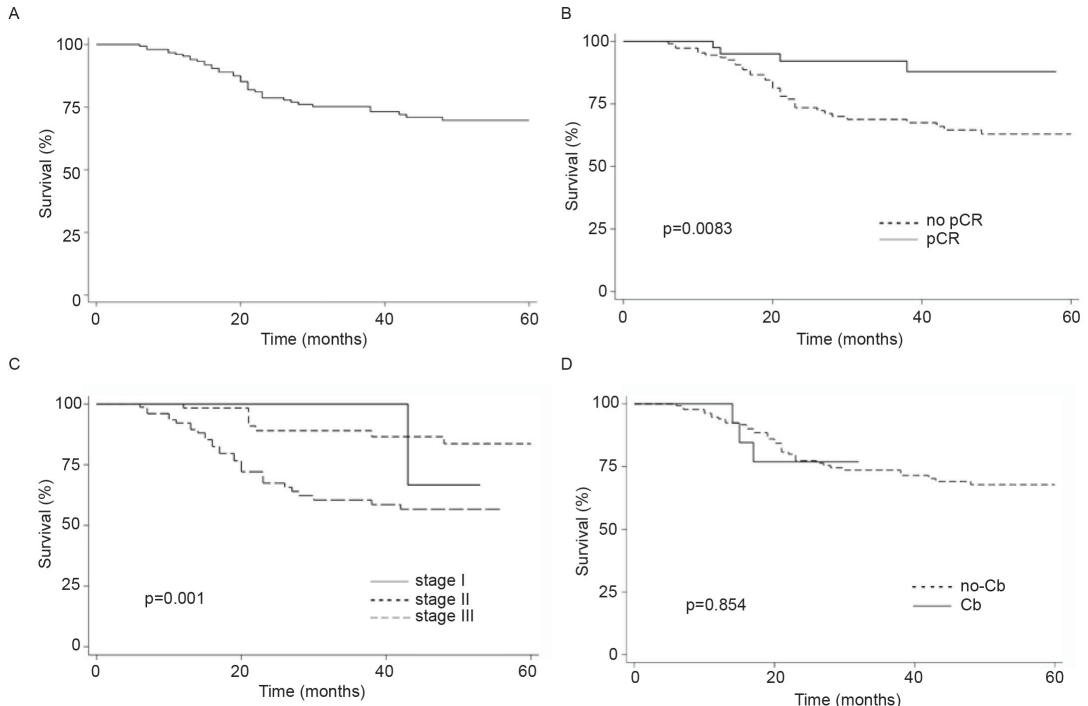

Figure 3. (a): DDFS in TNBC patients (b): by clinical stage, (c): presence/absence of pCR or (d) carboplatin (Cb) use.

\section{Discussion}

Every year, an estimated 5,500 new cases of BC are diagnosed in Chile [1]. From these, approximately 600 correspond to TNBC. Within this subset, the vast majority (92\%) are early stage cases [5]. In 2016, the Chilean a BC Ministry of Health implemented the GES (Explicit Guarantees in Health) programme that guarantees the access and opportunity to receive treatment to patients with suspected and/or diagnosed BC. However, GES does not include Cb for localised TNBC [13]. The authors' study provides real world evidence, seeking to contribute in the discussion of adding $\mathrm{Cb}$ as part of the standard neoadjuvant treatment for TNBC patients.

The authors' findings indicate a significant relationship between pCR and survival, confirming previous reports in TNBC [7]. Although the addition of $\mathrm{Cb}$ was associated with a discrete increase in $\mathrm{PCR}$, it was not associated with better survival. This could be explained by a combination of relatively small numbers of patients and low levels of pCR in the authors' cohort. Also, the addition of platinum in a subset of patients could have been part of a 'salvaging' strategy for those with a poor initial response to conventional therapies (without platinum compounds). Thus, obviously these patients had worse prognosis. Still, patients who received anthracycline plus taxane regimens displayed a clear trend towards increased pCR compared to first or second generation regimens, with or without $\mathrm{Cb}$ [14].

A number of prospective studies have evaluated the role of platinum based NACT in TNBC (shown in Table 2). Firstly, the Spanish group GEICAM [15] compared the effect of Doxorubicin plus Cyclophosphamide and Paclitaxel (ACT) with or without Cb in TNBC and found no differences. This study defined TNBC by cytokeratin 5/6+ IHC and/or HER1-positivity; pCR (ypTONO) was 30\% in both groups. Second, the CALGB 40603/Alliance [16] study randomised patients undergoing paclitaxel-based CT into two arms, with or without Cb followed by AC. This study found a significant benefit in pCR for the platinum group (54\% versus $41 \%$ ). In line with the authors' findings, pCR was associated with better OS and DFS regardless of treatment [17]. Third, the GeparSixto trial [18] randomised patients treated with paclitaxel plus liposomal doxorubicin and bevacizumab into placebo or $\mathrm{Cb}$ arms. In this case, pCR rates were 37\% versus 53.2\% $(p=0.005)$, respectively. Forty-seven month follow-up $[16,19]$ showed a $10 \%$ benefit in 3-year DFS (HR 0.56, 95\% Cl: 0.34-0.93; $p=0.02$ ) for the platinum group, but no benefit in OS. Importantly, the initial design of this trial was not powered for survival analysis and no alkylating drugs were included in either group. This study also included a subgroup analysis by BRCA mutational status and Homologous Recombination Deficiency (HRD) as biomarkers of platinum-response, confirming only HRD as a predictor of response. Finally, the BrighTNess was a multicenter randomised phase III study 
[20] that compared three NACT arms: T-AC (Paclitaxel weekly for 12 followed by 4 cycles of doxorubicin and cyclophosphamide), plus the combination of veliparib (a poly adenosine ribose polymerase inhibitor (PARPi)) and $\mathrm{Cb}$ (TVCb-AC) or Cb only (TCb-AC). Patients were stratified by BRCA mutational status. This study confirmed a pCR benefit given by $\mathrm{Cb}: 58 \%$ in the TCb-AC group, $31 \%$ in the T-AC group and $53 \%$ with TVCb-AC $(p<0.001)$. Consequently no benefit was observed by the use of PARPi, nor with $\mathrm{Cb}$ in BRCA mutant or wild type patients.

Table 2. Results of phase II-III clinical trials evaluating the addition of Cb to NACT schemes in TNBC. Literature review, last 10 years.

\begin{tabular}{|c|c|c|c|c|c|c|c|c|}
\hline Study type & $n$ & CT schedule & $\begin{array}{c}n \\
\text { arm }\end{array}$ & $\begin{array}{c}\text { pCR\% } \\
\text { ypTONO }\end{array}$ & $\begin{array}{c}p \\
\text { value }\end{array}$ & $\begin{array}{c}\text { DFS } \\
\mathrm{HR}(\mathrm{Cl})\end{array}$ & $\begin{array}{c}\text { OS } \\
\mathrm{HR}(\mathrm{Cl}) \\
\end{array}$ & Observations \\
\hline \multicolumn{9}{|l|}{ PHASE II } \\
\hline \multirow{2}{*}{$\begin{array}{l}\text { Alba et al [15] } \\
\text { GEICAM/2006-03 } \\
2012\end{array}$} & \multirow[t]{2}{*}{94} & $E C-D+C^{a}$ & 48 & 29.8 & \multirow[t]{2}{*}{0.61} & \multirow[t]{2}{*}{-} & \multirow[t]{2}{*}{-} & \multirow[t]{2}{*}{-} \\
\hline & & EC-D & 46 & 30.5 & & & & \\
\hline \multirow{2}{*}{$\begin{array}{l}\text { Ando et al [21] } \\
2014\end{array}$} & \multirow[t]{2}{*}{75} & TCb-FEC ${ }^{b}$ & 37 & $061 a$ & \multirow[t]{2}{*}{0.003} & \multirow[t]{2}{*}{-} & \multirow[t]{2}{*}{-} & \multirow[t]{2}{*}{ Subgroup analysis for TN } \\
\hline & & T-FEC & 38 & 26 & & & & \\
\hline \multirow{2}{*}{$\begin{array}{l}\text { Von Minckwitz et } \\
\text { al [17] GeparSixto } \\
2014\end{array}$} & \multirow[t]{2}{*}{315} & $\mathrm{TLA} \mathrm{Cb}+\mathrm{B}^{\mathrm{c}}$ & 158 & $53^{a}$ & \multirow[t]{2}{*}{0.005} & \multirow{2}{*}{$\begin{array}{c}0.56^{\mathrm{a}} \\
(0.34-0.93)\end{array}$} & \multirow{2}{*}{$\begin{array}{c}0.60 \\
(0.32-1.12)\end{array}$} & \multirow{2}{*}{$\begin{array}{l}47 \text { months follow up showed } \\
\text { increase in DFS for } \mathrm{Cb} \text { use with no } \\
\text { differences regarding BRCA status }\end{array}$} \\
\hline & & $T L A+B$ & 157 & 37 & & & & \\
\hline \multirow{3}{*}{$\begin{array}{l}\text { Sikov et al [17] } \\
\text { CALGB/ } \\
\text { ALLIANCE } 2016\end{array}$} & \multirow[t]{3}{*}{218} & $\mathrm{TCb}-\mathrm{AC}^{\mathrm{d}}$ & 111 & $54^{a}$ & \multirow[t]{3}{*}{0.003} & \multirow{3}{*}{$\begin{array}{c}0.84 \\
(0.58-1.22)\end{array}$} & \multirow{3}{*}{$\begin{array}{c}1.15 \\
(0.74-1.79)\end{array}$} & \multirow{3}{*}{$\begin{array}{l}39 \text { months follow up showed no } \\
\text { survival benefits for } \mathrm{Cb} \text { group. } \\
\text { Improved OS for } \mathrm{pCR}\end{array}$} \\
\hline & & $\begin{array}{l}\text { TCb-AC } \\
\text { TB-AC }\end{array}$ & & & & & & \\
\hline & & T-AC & 107 & 41 & & & & \\
\hline \multirow{2}{*}{$\begin{array}{l}\text { Zhang et al [34] } \\
2016\end{array}$} & \multirow[t]{2}{*}{91} & $\mathrm{TCb}^{\mathrm{e}}$ & 47 & $38.6^{\mathrm{a}}$ & \multirow[t]{2}{*}{0.014} & \multirow[t]{2}{*}{-} & \multirow[t]{2}{*}{-} & \multirow{2}{*}{$\begin{array}{l}55 \text { months follow up showed a } \\
21 \% \text { benefit in } 5 \text { years DFS with Cb } \\
(p=0.043)\end{array}$} \\
\hline & & ET & 44 & 14 & & & & \\
\hline \multirow{2}{*}{$\begin{array}{l}\text { Gluz et al [35] } \\
\text { WSG-ADAPT-TN } \\
2018\end{array}$} & \multirow[t]{2}{*}{336} & $\mathrm{Nab}-\mathrm{P}+\mathrm{Cb}^{f}$ & 154 & $45.9^{\mathrm{a}}$ & \multirow[t]{2}{*}{0.002} & - & - & No anthracyclines used \\
\hline & & Nab-P+Gem & 182 & 27.8 & & & & \\
\hline PHASE III & & & & & & & & \\
\hline Loibl et al [20] & 318 & $\mathrm{TCb}-\mathrm{AC}^{\mathrm{g}}$ & 160 & $58^{\mathrm{a}}$ & 0.0001 & - & - & No differences for BRCA status \\
\hline BrighTNess & & TCbV-AC & & & & & & \\
\hline 2018 & & $\mathrm{~T}-\mathrm{AC}$ & 158 & 31 & & & & \\
\hline $\begin{array}{l}\text { Schmid et al [27] } \\
\text { Keynote } 522\end{array}$ & 1,174 & $\mathrm{TCb}-\mathrm{AC}+\mathrm{Pem}$ & 784 & 65 & 0.0001 & $\begin{array}{c}0.63^{a} \\
(0.43-0.93)\end{array}$ & - & Both arms used $\mathrm{Cb}$ \\
\hline 2020 & & $\mathrm{TCb}-\mathrm{ACg}$ & 390 & 51 & & & & \\
\hline
\end{tabular}

TN; Triple negative, CT; Chemotherapy, AUC; Area under de curve, pCR; Pathological complete response, OS, Overall survival, DFS: Disease-free survival, DDFS; Distant disease-free survival, DHR; Deficient homologous recombination, E; Epirubicin, C; Cyclophosphamide, D; Docetaxel, T; Paclitaxel, B; Bevacizumab, Cb; Carboplatin, F; Fluorouracil, Nab-P; Nab-paclitaxel, G; Gemcitabine, V; Veliparib, Pem; Pembrolizumab, A; Doxorubicin, LA; non PEGylated liposomal doxorubicin ${ }^{\mathrm{a}} \mathrm{E} 90 \mathrm{mg} / \mathrm{m}^{2}+\mathrm{C} 600 \mathrm{mg} / \mathrm{m}^{2} \mathrm{q} 3 \mathrm{w} \times 4 \rightarrow \mathrm{D} 75 \mathrm{mg} / \mathrm{m}^{2}+\mathrm{Cb}$ AUC $(6 \mathrm{mg} / \mathrm{mL})$

'T $80 \mathrm{mg} / \mathrm{m}^{2} \mathrm{qw} \times 12+\mathrm{Cb}$ AUC $5 \mathrm{q} 3 \mathrm{w} \times 4 \rightarrow$ FEC: F $500 \mathrm{mg} / \mathrm{m}^{2}-\mathrm{E} 100 \mathrm{mg} / \mathrm{m}^{2}-\mathrm{C} 500 \mathrm{mg} / \mathrm{m}^{2} \mathrm{q} 3 \mathrm{w} \times 4$

cT $80 \mathrm{mg} / \mathrm{m}^{2} \mathrm{qw}+\mathrm{LA} 20 \mathrm{mg} / \mathrm{m}^{2} \mathrm{qw}+/-$ Cb AUC $2 \mathrm{qw} \times 18 \mathrm{w}+\mathrm{B} 15 \mathrm{mg} / \mathrm{kg} \mathrm{q} 3 \mathrm{w} \times 6$

dT $80 \mathrm{mg} / \mathrm{m}^{2} \mathrm{qw} \times 12+/-$ Cb AUC6 q3w $\times 4 \rightarrow$ A $60 \mathrm{mg} / \mathrm{m}^{2}+\mathrm{C} 600 \mathrm{mg} / \mathrm{m}^{2} \mathrm{q} 2 \mathrm{w} \times 4$

eT $175 \mathrm{mg} / \mathrm{m}^{2}$ on day $1+\mathrm{Cb}$ AUC5 on day 2 (q3w) or E $75 \mathrm{mg} / \mathrm{m}^{2}$ on day 1

${ }^{\mathrm{f}} \mathrm{A}$ Nab-T $125 \mathrm{mg} / \mathrm{m}^{2}+\mathrm{Cb}$ AUC2 day $1,8 \mathrm{q} 3 \mathrm{w}$ or G 1,000 mg/m² $\mathrm{dL}$, 8 three times weekly (q3w)

gT $80 \mathrm{mg} / \mathrm{m}^{2} \mathrm{qw} \times 12+/-$ Cb AUC6 q3w $\times 4 \rightarrow$ A $60 \mathrm{mg} / \mathrm{m}^{2}+\mathrm{C} 600 \mathrm{mg} / \mathrm{m}^{2} \mathrm{q} 2-3 \mathrm{w} \times 4$

hT $80 \mathrm{mg} / \mathrm{m}^{2} \mathrm{qw} \times 12+\mathrm{Cb}$ AUC6 q3w $\times 4 \rightarrow \mathrm{A} 60 \mathrm{mg} / \mathrm{m}^{2}+\mathrm{C} 600 \mathrm{mg} / \mathrm{m}^{2} \mathrm{q} 2-3 \mathrm{w} \times 4+/-$ Pem $200 \mathrm{mg}$ every 3 weeks $\times 8$ 
All studies demonstrated a significant increase in grade 3/4 toxicities by platinum compounds, mainly due to myelosuppression and neutropenia (56\%) and thrombocytopenia in $12 \%-20 \%$ of patients [16, 21], which leads to dose reduction in $68 \%$ of cases, and discontinuation in $48 \%$ [18]. In summary, phase $2 / 3$ and meta-analysis studies [9, 10] demonstrate that the addition of platinum compounds in NACT increases pCR rates in TNBC [22]. More recently, a meta-analysis [23] reported a significant OS benefit in various neoadjuvant-platinum trials.

Still, this lack of consistency in survival benefits for platinum based regimes could be attributed to several reasons. First, as explained above, this could be due to a lack of statistical power derived from small patient samples. Second, because of patient selection bias; where the addition of $\mathrm{Cb}$ in patients with initial good prognosis will not modify significantly survival rates. Third, obtaining pCR in some patients could be related to other factors (i.e., not caused by therapeutic interventions) such as increased lymphocytic infiltration [24] that determine a better overall prognosis, regardless of CT regimens. Therefore, pCR would be an intrinsic marker of good prognosis. Fourth, in some patients the increase of pCR could be at the expense of reducing minimal residual disease post-NACT. And minimal residual disease, at least theoretically, could have an equivalent prognosis to those with a ypTONO [25]. Thus, an increase in pCR in these cases would not be reflected in increased survival. Finally, the CREATE-X study [26] showed benefits in prolonging DFS and OS with adjuvant therapy in TNBC patients with residual disease, blurring an accurate assessment of the effect of pCR or Cb in NACT. Even more, recently published data from the KEYNOTE522 trial [27] demonstrated increases in PCR and DFS by neoadjuvant pembrolizumab in patients that received Cb.

This work has several limitations, mainly derived from its retrospective nature. First, only a small group received $\mathrm{Cb}$, thus lacking statistical power, second the authors' cohort contains a small proportion of stage I patients versus stage III, this could translate into fewer pCR despite the use of $\mathrm{Cb}$. Third, the authors' criteria for TNBC subtype classification was limited to IHC/FISH instead of a molecular analysis by gene microarray. In 2011, a study by Lehmann et al [28] postulated the existence of six subclasses within the TNBC subtype: Immunomodulatory, Mesenchymal, Mesenchymal stem-like, Luminal androgen receptor (LAIR), Unstable and two Basal-like subclasses (BL1 and BL2). More recently, Burstein et al [29] performed RNA and DNA profiling analysis and defined four TNBC subclasses or clusters: cluster 1: Luminal AR (AI), cluster 2: Mesenchymal, cluster 3: Basal-like immunosuppressed and cluster 4: Basal-like immune-activated. These classifications illustrate the high heterogeneity of TNBCs that could be reflected in different responses to NACT [30]. Fourth, the authors' study did not assess other potential biomarkers of platinum-response such as HRD, BRCA status or tumour infiltrating lymphocytes [31]. In this regard, the authors' study found only six BRCA1/2 mutants (3.8\%), much lower than the $20 \%$ reported in previous studies [32]. Finally, key information such as toxicity, ratio of chemotherapy delays, dose adjustments and suspension could not be obtained. Evidently, lower rates of CT completion or intensity could be a confounding factor in the lower rate of pCR. As described in the literature, reported toxicity should be critical in order to determine the true benefit of combined therapies.

\section{Conclusion}

In summary, this work provides relevant real-world evidence that might assist in decision-making and policies to incorporate the use of available drugs into clinical practice.

\section{Transparency section}

\section{Declaration of funding}

No sponsorship/funding.

\section{Author contributions}

BW, FA, LM, MLB, TM, MC, FD, MPP and CS were involved in the conception and design of the manuscript, and participated in the collection and interpretation of data. 
SMo, HG, BN, Cl, JM, SMu, JP, EK and MG made substantial contributions to the first draft and critically revised the final version of the manuscript.

All authors read and approved the final version of the manuscript to be published and are accountable for all aspects of this work.

\section{Acknowledgments}

No assistance in the preparation of this article is to be declared.

\section{Disclosure of interests}

$\mathrm{BN}$ is an advisor for Roche; JM is a consultant for Boehringer-Ingelheim and provides expert testimony to Roche; CS receives research funding from Roche and Novartis, and provides expert testimony for Pfizer and have received travel or accommodations, or expenses from Novartis and Pfizer; SMo is a consultant for Foundation Medicine; EK participates at the speakers' bureau for Novartis and have received travel support from Pfizer, Novartis and Roche Pharma; MG is an advisor for Merck Sharp \& Dohme and Novartis, participates at the speakers' bureau for Bristol-Myers \& Squibb and Bayer. Also receives research funding from Bristol-Myers \& Squibb (Inst) and Novartis (Inst). Also, has received travel accommodations and expenses from Roche. The funders had no role in the design of the study; in the collection, analyses or interpretation of data; in the writing of the manuscript, or in the decision to publish the results.

\section{References}

1. Cancer Today [Internet] Cancer Today (Washington, D.C.: National Academies Press) [https://gco.iarc.fr/today/fact-sheets-cancers]

2. DeSantis CE, Ma J, and Gaudet MM, et al (2019) Breast cancer statistics, 2019 CA Cancer J Clin [Internet] 69 438-451 https://doi. org/10.3322/caac.21583 PMID: 31577379

3. Keum N, Greenwood DC, and Lee DH, et al (2015) Adult weight gain and adiposity-related cancers: a dose-response meta-analysis of prospective observational studies [Internet] J Natl Cancer Inst [http://eprints.whiterose.ac.uk/84759/] https://doi.org/10.1093/jnci/ djv088

4. Lauby-Secretan B, Scoccianti C, and Loomis D, et al (2016) Body fatness and cancer - viewpoint of the IARC working group [Internet] N Engl J Med 375(8) 794-798 https://doi.org/10.1056/NEJMsr1606602 PMID: 27557308 PMCID: 6754861

5. Maiz C, Silva F, and Domínguez F, et al (2020) Mammography correlates to better survival rates in breast cancer patients: a 20-year experience in a University health institution Ecancermedicalscience 141005 https://doi.org/10.3332/ecancer.2020.1005 PMID: 32104207 PMCID: 7039691

6. Asselain B, Barlow W, and Bartlett J, et al (2018) Long-term outcomes for neoadjuvant versus adjuvant chemotherapy in early breast cancer: meta-analysis of individual patient data from ten randomised trials Lancet Oncol [Internet] [cited 2020 Dec 1] 19 27-39 [https:// www.ctsu.ox.ac.uk/] Date accessed: 1/12/20 https://doi.org/10.1016/S1470-2045(17)30777-5

7. Cortazar P, Zhang L, and Untch M, et al (2014) Pathological complete response and long-term clinical benefit in breast cancer: the CTNeoBC pooled analysis Lancet [Internet] 384 164-172 https://doi.org/10.1016/S0140-6736(13)62422-8 PMID: 24529560

8. NCCN International Programs [Internet] [https://www.nccn.org/global/?gclid=Cj0KCOjwvb75BRD1ARIsAP6LcquNhwD3vILLHJ3kYJc9 phumyt4ohivAVBwqQnWR6-jlpKnPRfDhQYQaAnObEALw_wcB]

9. Poggio F, Bruzzone M, and Ceppi M, et al (2018) Platinum-based neoadjuvant chemotherapy in triple-negative breast cancer: a systematic review and meta-analysis Ann Oncol 29(7) 1497-1508 https://doi.org/10.1093/annonc/mdy127 PMID: 29873695 
10. Wang D, Feng J, and Xu B (2019) A meta-analysis of platinum-based neoadjuvant chemotherapy versus standard neoadjuvant chemotherapy for triple-negative breast cancer [Internet] Futur Oncol 15(23) 2779-2790 https://doi.org/10.2217/fon-2019-0165

11. Allison $\mathrm{KH}$, Hammond $\mathrm{MEH}$, and Dowsett $\mathrm{M}$, et al (2020) Estrogen and progesterone receptor testing in breast cancer: ASCO/CAP guideline update J Clin Oncol [Internet] 38 1346-1366 [https://jhu.pure.elsevier.com/en/publications/estrogen-and-progesteronereceptor-testing-in-breast-cancer-ascoc] https://doi.org/10.1200/JCO.19.02309 PMID: 31928404

12. Hortobagyi GN, Connolly JL, and D'Orsi CJ, et al (2017) Breast AJCC Cancer Staging Man [Internet] (Cham: Springer International Publishing) pp 589-636 [https://cancerstaging.org/references-tools/deskreferences/Documents/AJCC Breast Cancer Staging System.pdf]

13. Castillo C del SM, Cabrera MEC, and Derio PL, et al (2017) Resultados del tratamiento del cáncer de mama, programa nacional de cáncer del adulto Rev Med Chil 145 1507-1513 https://doi.org/10.4067/s0034-98872017001201507

14. Anampa J, Makower D, and Sparano JA (2015) Progress in adjuvant chemotherapy for breast cancer: an overview [Internet] BMC Med p 195 Date accessed: 1/12/20 https://doi.org/10.1186/s12916-015-0439-8

15. Alba E, Chacon Jl, and Lluch A, et al (2012) A randomized phase II trial of platinum salts in basal-like breast cancer patients in the neoadjuvant setting. Results from the GEICAM/2006-03, multicenter study Breast Cancer Res Treat [Internet] 136 487-493 https://doi. org/10.1007/s10549-012-2100-y PMID: 23053638

16. Loibl S, Weber KE, and Timms KM, et al (2018) Survival analysis of carboplatin added to an anthracycline/taxane-based neoadjuvant chemotherapy and HRD score as predictor of response-final results from GeparSixto Ann Oncol 29 2341-2347 https://doi. org/10.1093/annonc/mdy460 PMID: 30335131

17. Sikov W, Berry D, and Perou C, et al (2016) Abstract S2-05: event-free and overall survival following neoadjuvant weekly paclitaxel and dose-dense AC +/- carboplatin and/or bevacizumab in triple-negative breast cancer: outcomes from CALGB 40603 (Alliance) Cancer Res American Association for Cancer Research (AACR) pp S2-05 [https://institutionalrepository.aah.org/onc/70]

18. Von Minckwitz G, Schneeweiss A, and Loibl S, et al (2014) Neoadjuvant carboplatin in patients with triple-negative and HER2-positive early breast cancer (GeparSixto; GBG 66): a randomised phase 2 trial Lancet Oncol 15 747-756 [https://pubmed.ncbi.nlm.nih. gov/24794243/] https://doi.org/10.1016/S1470-2045(14)70160-3

19. von Minckwitz G, Loibl S, and Schneeweiss A, et al (2016) Abstract S2-04: early survival analysis of the randomized phase II trial investigating the addition of carboplatin to neoadjuvant therapy for triple-negative and HER2-positive early breast cancer (GeparSixto) Cancer Res [Internet] American Association for Cancer Research (AACR) S2-04 [https://cancerres.aacrjournals.org/content/76/4_Supplement/S2-04]

20. Loibl S, O'Shaughnessy J, and Untch M, et al (2018) Addition of the PARP inhibitor veliparib plus carboplatin or carboplatin alone to standard neoadjuvant chemotherapy in triple-negative breast cancer (BrighTNess): a randomised, phase 3 trial Lancet Oncol [Internet] 19 497-509 https://doi.org/10.1016/S1470-2045(18)30111-6 PMID: 29501363

21. Ando M, Yamauchi H, and Aogi K, et al (2014) Randomized phase Il study of weekly paclitaxel with and without carboplatin followed by cyclophosphamide/epirubicin/5-fluorouracil as neoadjuvant chemotherapy for stage II/IIIA breast cancer without HER2 overexpression Breast Cancer Res Treat [Internet] 145 401-409 https://doi.org/10.1007/s10549-014-2947-1 PMID: 24728578

22. Dieci MV, Del Mastro L, and Cinquini M, et al (2019) Inclusion of platinum agents in neoadjuvant chemotherapy regimens for triple-negative breast cancer patients: Development of GRADE (grades of recommendation, assessment, development and evaluation) recommendation by the Italian association of medical onco Cancers (Basel) [Internet] 11(8) 1137 [https://pubmed.ncbi.nlm.nih. gov/31398896/] https://doi.org/10.3390/cancers11081137

23. Spring LM, Fell G, and Arfe A, et al (2020) Pathologic complete response after neoadjuvant chemotherapy and impact on breast cancer recurrence and survival: a comprehensive meta-analysis Clin Cancer Res [Internet] 26 2838-2848 https://doi.org/10.1158/1078-0432. CCR-19-3492 PMID: 32046998 PMCID: 7299787 
24. Denkert C, von Minckwitz G, and Darb-Esfahani S, et al (2018) Tumour-infiltrating lymphocytes and prognosis in different subtypes of breast cancer: a pooled analysis of 3771 patients treated with neoadjuvant therapy Lancet Oncol [Internet] 19 40-50 [https://pubmed. ncbi.nlm.nih.gov/29233559/] https://doi.org/10.1016/S1470-2045(17)30904-X

25. Symmans WF, Wei C, and Gould R, et al (2017) Long-term prognostic risk after neoadjuvant chemotherapy associated with residual cancer burden and breast cancer subtype J Clin Oncol [Internet] 35 1049-1060 https://doi.org/10.1200/JCO.2015.63.1010 PMID: 28135148 PMCID: 5455352

26. Masuda N, Lee SJ, and Ohtani S, et al (2017) Adjuvant capecitabine for breast cancer after preoperative chemotherapy N Engl J Med [Internet] 376 2147-2159 https://doi.org/10.1056/NEJMoa1612645 PMID: 28564564

27. Schmid P, Cortes J, and Pusztai L, et al (2020) Pembrolizumab for early triple-negative breast cancer N Engl J Med [Internet] 382810 821 https://doi.org/10.1056/NEJMoa1910549 PMID: 32101663

28. Lehmann BD, Bauer JA, and Chen X, et al (2011) Identification of human triple-negative breast cancer subtypes and preclinical models for selection of targeted therapies J Clin Invest [Internet] 121 2750-2767 https://doi.org/10.1172/JCI45014 PMID: 21633166 PMCID: $\underline{3127435}$

29. Burstein MD, Tsimelzon A, and Poage GM, et al (2015) Comprehensive genomic analysis identifies novel subtypes and targets of triple-negative breast cancer Clin Cancer Res [Internet] 21 1688-1698 [https://pubmed.ncbi.nlm.nih.gov/25208879/] https://doi. org/10.1158/1078-0432.CCR-14-0432 PMCID: 4362882

30. Santonja A, Sánchez-Muñoz A, and Lluch A, et al (2018) Triple negative breast cancer subtypes and pathologic complete response rate to neoadjuvant chemotherapy Oncotarget [Internet] 9 26406-26416 https://doi.org/10.18632/oncotarget.25413 PMID: 29899867 PMCID: 5995183

31. Bernardi R and Gianni L (2014) Hallmarks of triple negative breast cancer emerging at last? Cell Res [Internet] 24 904-905 https://doi. org/10.1038/cr.2014.61 PMID: 24810303 PMCID: 4123289

32. Gonzalez-Angulo AM, Timms KM, and Liu S, et al (2011) Incidence and outcome of BRCA mutations in unselected patients with triple receptor-negative breast cancer Clin Cancer Res [Internet] 17 1082-1089 https://doi.org/10.1158/1078-0432.CCR-10-2560 PMID: 21233401 PMCID: 3048924 